\title{
Construing the communication between powerhouse and ri- bosomal factory of cell: A small molecular viscometer comes to the rescue
}

\author{
Tanoy Dutta, ${ }^{1 \#}$ Sreeparna Das, ${ }^{\text {\# }}$ Ishaan Gupta, ${ }^{2}$ and Apurba Lal Koner ${ }^{* 1}$ \\ ${ }^{1}$ Bionanotechnology Laboratory, Department of Chemistry, Indian Institute of Science Education and Research Bho- \\ pal, Bhopal Bypass Road, Bhauri, Bhopal, Madhya Pradesh- 462066, INDIA \\ ${ }^{2}$ Department of Biochemical Engineering and Biotechnology, Indian Institute of Technology Delhi, Hauz Khas, New \\ Delhi- 110016, INDIA
}

KEYWORDS. Mitochondria; Molecular rotor; Nucleolus; Organelle communication; Metaxin protein

\begin{abstract}
Fluorescent probes for specific inter-organelle communication are of massive significance as such communication is essential for a diverse range of cellular events. Here, we present the microviscosity-sensitive fluorescence marker, Quinaldine red (QR), and its dual organelle targeting light-up response in live cells. This biocompatible probe was able to localize in mitochondria and nucleolus simultaneously. While $\mathbf{Q R}$ was able to sense the viscosity change inside these compartments under the induced effect of an ionophore and ROS-rich microenvironment, the probe's ability to stain mitochondria remained unperturbed even after protonophore-induced depolarization. Consequently, a systematic quantification was performed to understand the alteration of microviscosity. Similar behavior in two distinct organelles implied that QR binds to Metaxin-2 protein, common to mitochondrial and nucleolar proteomes. We believe this is the first of its kind investigation that identifies the inter-organelle communications marker and opens up a new dimension in this field.
\end{abstract}

\section{INTRODUCTION}

Cellular organelles, since their discovery, are primarily considered as individual entities of particular composition that are entitled to specific roles and functions to implement several intra-cellular events. Nevertheless, such solitary roles are no longer pertinent as defied by the recent scientific advancements on inter-organelle communication. ${ }^{1-4}$ Organelles exchange information via certain biomolecules and metabolites to maintain long-term cellular homeostasis through different modes of communication: inter-organelle signaling, intracellular trafficking, membrane contact sites (MCS), and inter-organelle transient connections. ${ }^{3,5}$ In terms of organelle connectivity, mitochondria have remained in the center stage of communication being well-connected to the endoplasmic reticulum, lipid droplets, peroxisomes, and nucleus. Such mitochondria-centric communication is responsible for calcium signaling and transport, phospholipid synthesis and transfer, maintaining lipid balance through $\beta$-oxidation, and transfer of noncoding RNA. ${ }^{6-7}$ In this contribution, we took a specific interest to explore the communication between mitochondria and nucleolus using a molecular viscometer as a red fluorogenic reporter. Upon investigation, it becomes apparent that the fluorescent signal acts as a tool to quantify the effect in both mitochondria and nucleolus compartments in certain homeostatic and non-homeostatic conditions.
Mitochondria, often referred to as the 'powerhouse of the cell', are present in the cytoplasm of most of the eukaryotes. These unusual membrane-bound organelles primarily produce cellular energy, participate in metabolic activities, while also participating in cell signaling, production of heat, and mediating cell differentiation and death. ${ }^{8-9} \mathrm{Mi}-$ tochondria, owing to their tendency for aberrant free-electron release, remain a major site for ROS generation..$^{10-15}$ Despite the presence of its inbuilt ROS scavenging system, often an antioxidant protein, sometimes they cause damages to mtDNA. Additionally, the production of ROS and related oxidative stress alters microenvironmental viscosity and polarity at a specific region. ${ }^{16}$ Retarded mitochondrial respiration and metabolism, a result of increased viscosity attributable to dysfunctional biomolecular interactions and metabolite diffusion, subsequently adds on to cell death. ${ }^{17}$ Mitochondrial dysfunction is also implicated in several neurodegenerative diseases e.g. Alzheimer's disease and Parkinson's disease. ${ }^{12}$ Although protein crosslinking by ROS and subsequent formation of protein aggregates are suspected to be the main factor for increasing microenvironmental viscosity, the exact evidence remains unclear. $^{18}$ Therefore, monitoring mitochondrial viscosity during various biological processes proves to be of enormous importance in providing valuable insight into disease surveillance, thus making the evolution of simpler mitochondria-targeted precise fluorescent probes crucial. Investigation of mitochondrial viscosity copiously utilizes 
fluorescent probes working on the molecular rotor principle. ${ }^{12,17}$ These are $\pi$-conjugated fluorescent molecules, usually containing electron donor and acceptor moieties connected via a $\pi$-bridge/flexible linker. ${ }^{19}$ Intramolecular free rotation of the connected moieties (often termed as 'rotators'), around the $\pi$-conjugated bridge in a low-viscous environment, induces non-radiative thermal relaxation of excitation energy and quenching of fluorescence while inhibition of the rotation in high viscous media restores the fluorescence. ${ }^{20-21}$

Hydrophobicity of the inner mitochondrial membrane along with a negative membrane potential has led to a mitochondria-targeted probe being entitled to some specific structural features. ${ }^{22}$ The most popular mitochondria targeting moieties are lipophilic cations, triphenylphosphonium (TPP) being the most celebrated example. The TPP cation has been attached to a series of moieties e.g., branched polymer, ${ }^{23}$ dendrimer, ${ }^{24}$, and small molecules such as $\alpha$-tocopherol,,${ }^{25}$ peroxidase mimics, ${ }^{26}$ and coenzyme $\mathrm{Q},{ }^{27}$ to make them suitable for delivery to mitochondria. ${ }^{111}{ }^{17}$ Rhodamine, ${ }^{28}$ BODIPY, ${ }^{29}$ indoline, ${ }^{30-31}$ and julolidine ${ }^{32}$ derivatives are some other members to name from the same group. Commercially available MitoTracker dyes possess a delocalized positive charge to assist their plasma membrane potential-dependent accumulation in mitochondria. Besides mapping mitochondrial viscosity, some of these molecules were found useful in probing membrane potential ${ }^{33}$ and ROS inside these organelles. ${ }^{11}$ Despite the evolution of numerous mitochondria targeting molecular probes, the discovery of selective probes remains challenging, as we extend our understanding and unfold the function and involvement of the organelle in several diseases and health conditions.

The nucleolus, known as the 'ribosomal factory of the cell', is one of the most conspicuous and distinct membraneless substructures inside the nucleus primarily involved in ribosome biogenesis and monitoring proteins for cell cycle

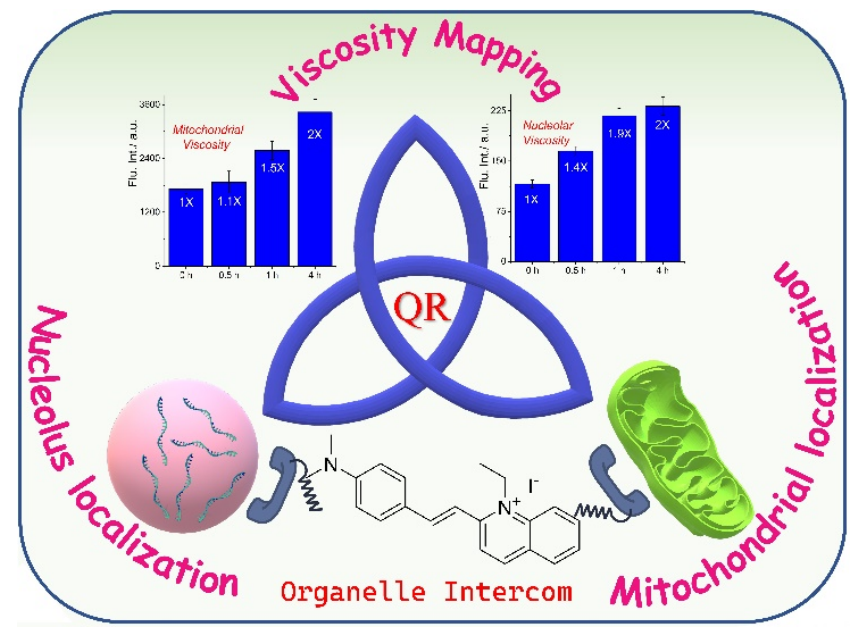

Scheme 1. Schematic representation of the present work involving simultaneous dual organelle localization and viscosity mapping using Quinaldine Red $(\mathbf{Q R})$ as a fluorescent probe. regulation. It is a dynamic entity with a highly dense domain as compared to the surrounding nucleoplasm. ${ }^{34}$ Mainly, it consists of three distinct structural componentsfibrillar center, dense fibrillar component, and granular center. The nucleolus is also involved in cell cycle regulation, growth, and development. ${ }^{35}$ However, little is known about the microviscous response of nucleolus during intracellular events. The probe of interest, $\mathbf{Q R}$ is also capable of staining nucleolus alongside mitochondria owing to the interaction with a protein, namely metaxin-2. This dual organelle localization brings a new dimension to study the synchronous behavior of the corresponding cellular compartments during non-homeostatic events such as induced stress and apoptosis, arrested cell cycle checkpoints. With this report, we introduce a well-known indicator dye, $\mathbf{Q R}$, as an efficient fluorescent viscometer and aim to invigorate the field of inter-organelle communication (Scheme 1). For this study, a small molecular organic dye, $\mathbf{Q R}$ was selected having an electron-donating N, N-dimethylaminophenyl group connected to an electron-deficient quaternized quinoline moiety through a $\mathrm{C}-\mathrm{C}$ double bond as depicted in the FMO picture (Figure S1). QR, a dark red solid, has been proved useful as an indicator, ${ }^{36-37}$, and a fluorescent tag. ${ }^{38}$ The molecule is biocompatible, easily synthesized, and shows excellent fluorescence response upon binding with a protein or nucleic acid. The electron-withdrawing quinoline moiety present in the molecule was hypothesized to be the key aspect for its targeting ability towards mitochondria.

Thus, it becomes clear from the above facts that the quaternized nitrogen present in the dye may interact with the phospholipids of the mitochondrial membrane and although the aggregated state of the molecule may experience some change, ${ }^{39}$ the targeting ability of the molecule would experience little to no change as $\mathrm{pH}$ gradient across the membrane is affected or least destroyed. At the outset, we became inquisitive to examine the photo-physical behavior, viscosity-dependent emission enhancement of the dye, and understand its usability. The dye working on the molecular rotor principle showed 57 -fold fluorescence emission enhancement as viscosity increased from 1 to $480 \mathrm{cP}$. Also, notable was its excellent sensitivity as a response to the change in viscosity and photostability. The probe was found to be non-cytotoxic, and it selectively localizes in mitochondria, simultaneously staining the nucleolus. The probe, based on its optical response, can distinguish between the homeostatic and induced viscosity state of the mitochondrial matrix of living cells upon various subcellular stress conditions.

\section{RESULTS AND DISCUSSION}

QR as a molecular viscometer. The spectroscopic pointof-view. The lipophilicity of any probe, that is being anticipated to localize in the mitochondria, ${ }^{40}$ is one of the most important physicochemical parameters to look into. The octanol-water partition coefficient is the commonest way to express the lipophilicity of a compound. ${ }^{41}$ It is defined as 
the ratio of the concentration of a solute in a water-saturated octanol phase to its concentration in an octanol-saturated aqueous phase. Now, the model of the structure-activity relation specifying a physicochemical parameter of a probe staining mitochondria should follow the criteria of electric charge, $\mathrm{Z}>\mathrm{o}$ and $\mathrm{o}<\log \mathrm{P}<+5$, $\log \mathrm{P}$ being the
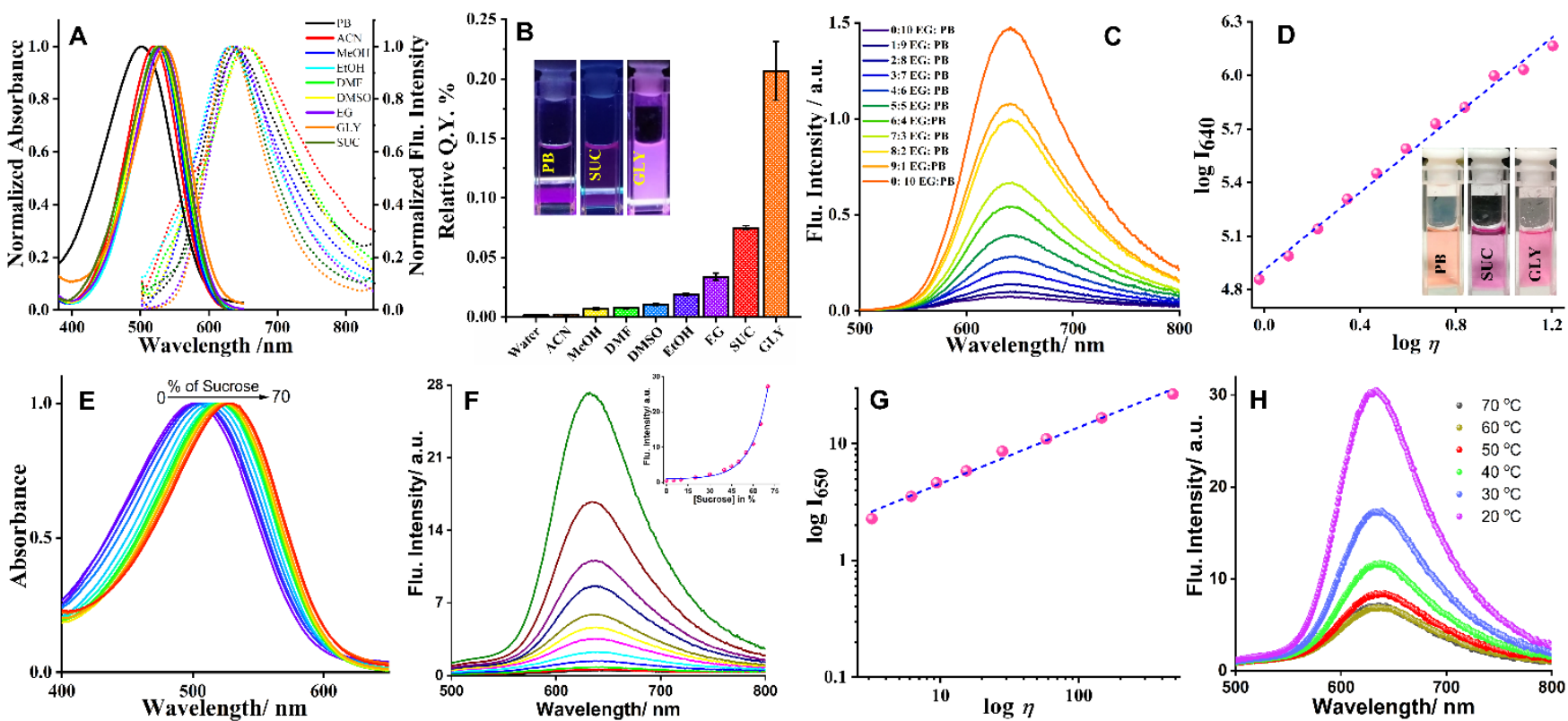

Figure 1. (A) Normalized absorption and emission spectra of $\mathbf{Q R}$ in different solvents, (B) relative quantum yield of $\mathbf{Q R}$ in different solvents, (C) emission spectra of $10 \mu \mathrm{M}$ QR in Ethylene glycol (GLY) / phosphate buffer (PB) mixture of varying ratios, (D) plot of $\log \mathrm{I}_{640}$ and $\log \eta, \lambda_{\mathrm{ex} / \mathrm{em}}=48 \mathrm{o} / 640 \mathrm{~nm}$. Insets of (B) and (D) show representative images of $Q R$ solution in phosphate buffer, $70 \%$ sucrose $\left(\right.$ SUC), and glycerol under UV irradiation $\left(\lambda_{\mathrm{ex}}=365 \mathrm{~nm}\right)$ and daylight, respectively. (E) Absorption and (F) emission spectra of $10 \mu \mathrm{M}$ QR in aqueous sucrose solution of varying concentrations (o, 5, 10, 20, 30, 40, 45, 50, 55, 60, 65, 70\%), the inset shows the intensity of $\mathbf{Q R}$ with increasing percentage of sucrose, $(\mathbf{G})$ plot of $\log \mathrm{I}_{640}$ and $\log \eta, \lambda_{\mathrm{ex} / \mathrm{em}}=48 \mathrm{o} / 640 \mathrm{~nm},(\mathbf{H})$ emission spectra of $10 \mu \mathrm{M}$ QR in $70 \%$ aqueous sucrose solution with increasing temperature $\left(20,30,40,50,60,70{ }^{\circ} \mathrm{C}\right)$.

The aqueous solution of the probe, orange in color, showed an absorption peak centered at $500 \mathrm{~nm}$. While in highly viscous media the peak was shifted to $535 \mathrm{~nm}$ with a change of solution color to pink. A strong fluorescence peak centered at $640 \mathrm{~nm}$ was observed as the viscosity of the environment increased. The probe exhibited a good linear relationship $\left(R^{2}=0.99\right.$, with a slope of $\mathrm{x}=0.45, \eta$ in $\left.\mathrm{cP}\right)$ between the fluorescence intensity response $\left(\log \mathrm{I}_{640}\right)$ and viscosity $(\log \eta)$, by fitting the Förster-Hoffmann equation ${ }^{43}$ (Eqn. S1). Steady-state absorption and fluorescence spectra of QR were recorded in solvents of different polarities (Figure 1A) and the respective absorbance and intensities have been normalized at the wavelength of maxima. In the solvents of higher polarity e.g., DMF, a redshift of $\sim 25 \mathrm{~nm}$ is observed in the absorption band (w.r.t. water) recorded in solvents of different polarities (Figure $\mathbf{1 A}$ ), and the respective absorbance and intensities have been normalized at the wavelength of maxima. The shift of absorbance maxima (although very small, $\sim 10 \mathrm{~nm}$ ) in protic solvents follows the viscosity order of the solvents. The quantum yield of QR determined in various solvents again follows the order of increase of viscosity of the medium (Figure 1B, Table $S_{1}$ ). In solvents of low viscosity, quantum yield does not exceed 0.015; in highly viscous media as glycerol, the value reaches $0.21 \pm 0.03$ (Figure $\mathbf{1 B})$. In high polarity solvents such as acetonitrile and methanol, QR shows weak fluorescence. However, the emission maxima in polar aprotic solvents are centered around $650 \mathrm{~nm}$, whereas in a polar protic solvent the maxima are centered on $630 \mathrm{~nm}$. Thus, solvent polarity-dependent fluorescence emission, as well as quantum yield, suggests the involvement of several relaxation pathways. The broad nature of the absorbance and fluorescence emission further infers the presence of conformers with considerably broader distribution of the dihedral angles between the planes of the N, N-dimethylaniline, and the quinoline moieties in the $S_{1}$ state compared to the ground electronic $\left(\mathrm{S}_{\mathrm{o}}\right)$ state, i.e., loss of structural planarity of the excited state.

Unfolding the viscosity sensitivity of $Q R$. The viscositydependent spectroscopic property of $\mathbf{Q R}$ was investigated using various ratios of $\mathrm{PB}$ and ethylene glycol (EG). Upon increasing the EG fraction from o to 100, a 22 -fold enhancement of fluorescence was observed (Figure 1C). The aqueous solution of QR looks orange in color, whereas in GLY a pink solution was obtained (Inset, Figure $\mathbf{D D}$ ). The weak fluorescence intensity of $\mathbf{Q R}$ in water may be rationalized based on the free rotation of the quinoline and N, N-dimethylamino moieties, forming a twisted and non-radiative 

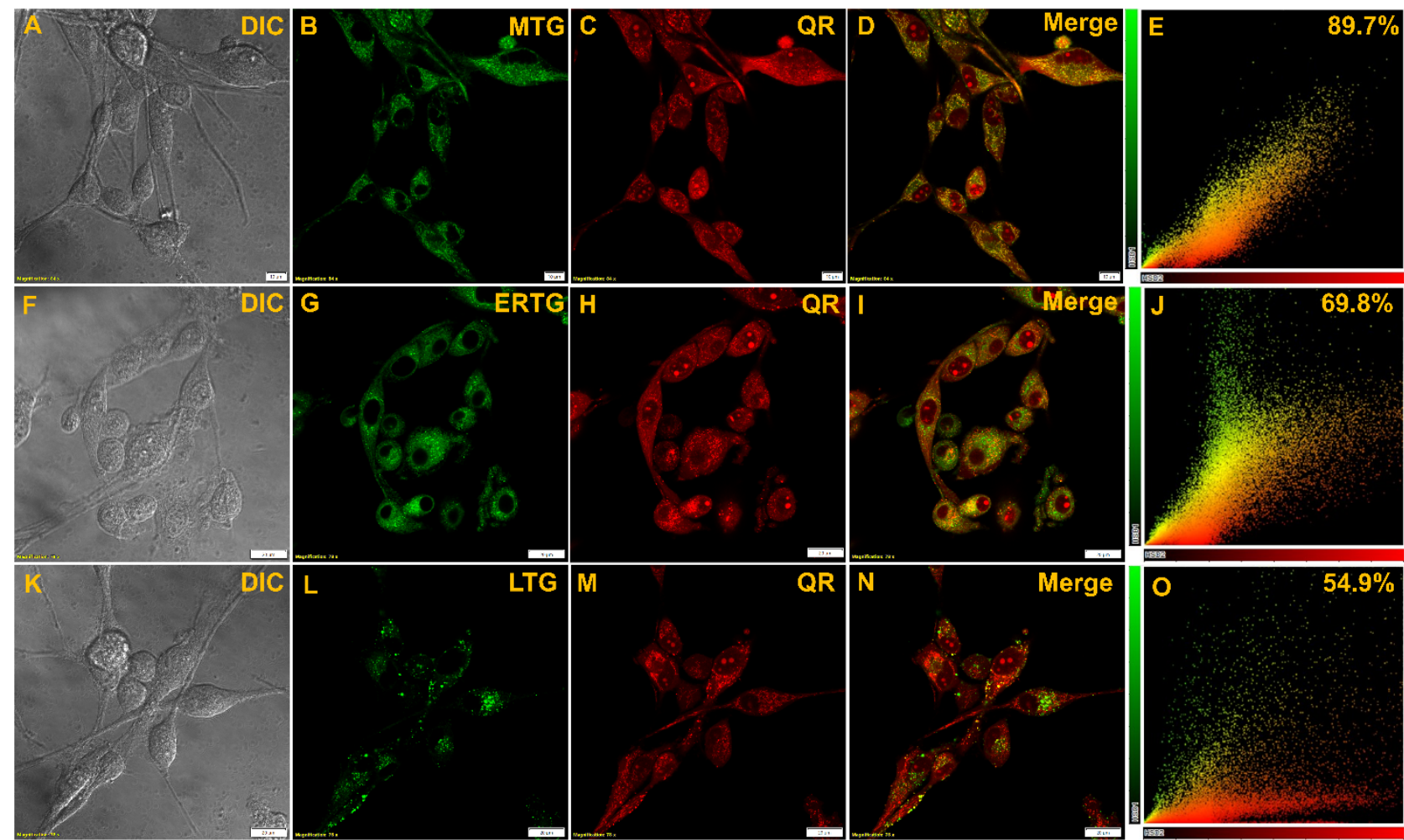

Figure 2. Confocal live-cell colocalization experiments in U-87 MG cells. (A), (F), and (K) show DIC images; (B), (G), and (L) show the FITC channel images stained with o.3 $\mu \mathrm{M}$ MitoTracker green, o.3 $\mu \mathrm{M}$ ER-Tracker Green, and LysoTracker Green; (C), $(\mathbf{H})$, and (M) show the TRITC channel images stained with $5 \mu \mathrm{M} \mathrm{QR;(D),} \mathrm{(I),} \mathrm{and} \mathrm{(N)} \mathrm{show} \mathrm{the} \mathrm{corresponding} \mathrm{merge} \mathrm{images;} \mathrm{(E),}$ $(\mathrm{J})$, and $(\mathbf{O})$ shows the scatter plot to get the respective Pearson's correlation coefficients. (Scale bar: $10 \mu \mathrm{m}$ ).

decayed excited state. A linear relationship $\left(R^{2}=0.99\right.$, slope $=1.08, \eta$ in $\mathrm{cP}$ ) was observed from the $\log \mathrm{I}$ vs. $\log \eta$ plot, by fitting the Förster-Hoffmann equation (Figure 1D, Eqn. S1). The fluorescent lifetime also was found to get enhanced with increasing viscosity (Figure $\mathbf{S 2 B}$ ); good linearity was observed from $\log \tau$ vs. $\log \eta$ plot in sucrose solutions with increasing viscosity (Figure $\mathbf{S 2} \mathbf{C}$ ). The $\mathrm{pH}$ response of $\mathbf{Q R}$, an important aspect of a probe for biological applications, was investigated from UV-Vis. spectroscopy. The absorption spectra of the dye showed typical $\mathrm{pH}$ response patterns of a $\mathrm{pH}$ indicator. An increase of the absorbance maxima centered at $500 \mathrm{~nm}$ was observed with an isosbestic point at $395 \mathrm{~nm}$, with a simultaneous decrease of a peak appearing at $360 \mathrm{~nm}$ (Figure $\mathbf{S}_{3}$ ). At lower $\mathrm{pH}$, the orange-red color of the dye disappeared and the solution turned colorless. The $\mathrm{p} K_{\mathrm{a}}$ value observed from the inflection point of the plot of absorbance at $\lambda_{\max } \mathrm{vs} \mathrm{pH}$ was close to $2.7 \cdot{ }^{37}$ In viscous medium e.g., 70\% sucrose, (Figure $\mathbf{1 E} \& \mathbf{1 F}$ ) bright pink solution of QR displayed a strong fluorescence at $635 \mathrm{~nm}$ with a 57 -fold increase (see inset of Figure $1 F$ ), due to limited free rotation. The emission peak in $70 \%$ sucrose was, however, narrower indicating a more constrained environment now being experienced by the chromophore. With an increase of viscosity from 1.1 to $480.6 \mathrm{cP}$ in different percentages of sucrose solutions (\% $\mathrm{w} / \mathrm{w})$ brilliant enhancement of fluorescence occurred. Good linearity $\left(R^{2}=0.99\right.$, slope $=0.45, \eta$ in $\left.\mathrm{cP}\right)$ observed in the viscosity range (Figure $\mathbf{1 G}$ ) further validates the utility of the probe for the detection of viscosity in various media, including biological systems. To further validate the viscosity-induced emission enhancement, temperature-dependent emission spectra were recorded with the $70 \%$ aqueous solution of sucrose (10 $\mu \mathrm{M}$ in QR). With an increase in temperature, viscosity decreases as the greater thermal energy allow the molecules to overcome intermolecular forces responsible for generating viscous forces across layers in a liquid. Figure $\mathbf{1 H}$ shows a decrease of fluorescence intensity as temperature increases, however, no change of emission is observed beyond $70{ }^{\circ} \mathrm{C}$.

Intracellular dual organelle localization of QR. As evident from the detailed spectroscopic studies, the viscosity-based fluorometric response of QR prompted us for live-cell imaging experiments for intracellular viscosity measurements. Initially, the biocompatibility of the probe was monitored by the standard MTT assay in U-87 MG glioblastoma cell lines. Following a $24 \mathrm{~h}$ incubation with different concentrations of $Q R$ in live $\mathrm{U}-87 \mathrm{MG}$ cells, the $\mathrm{IC}_{50}$ value was found to be above $35 \mu \mathrm{M}$ (Figure $\mathbf{S}_{4}$ ). Therefore, for live-cell imaging studies, $5 \mu \mathrm{M}$ QR was used having $\sim 80 \%$ viable cells. Confocal microscopy results suggested the localization of $\mathbf{Q R}$ in the mitochondrial compartments. To confirm, commercially available Mitotracker green was 

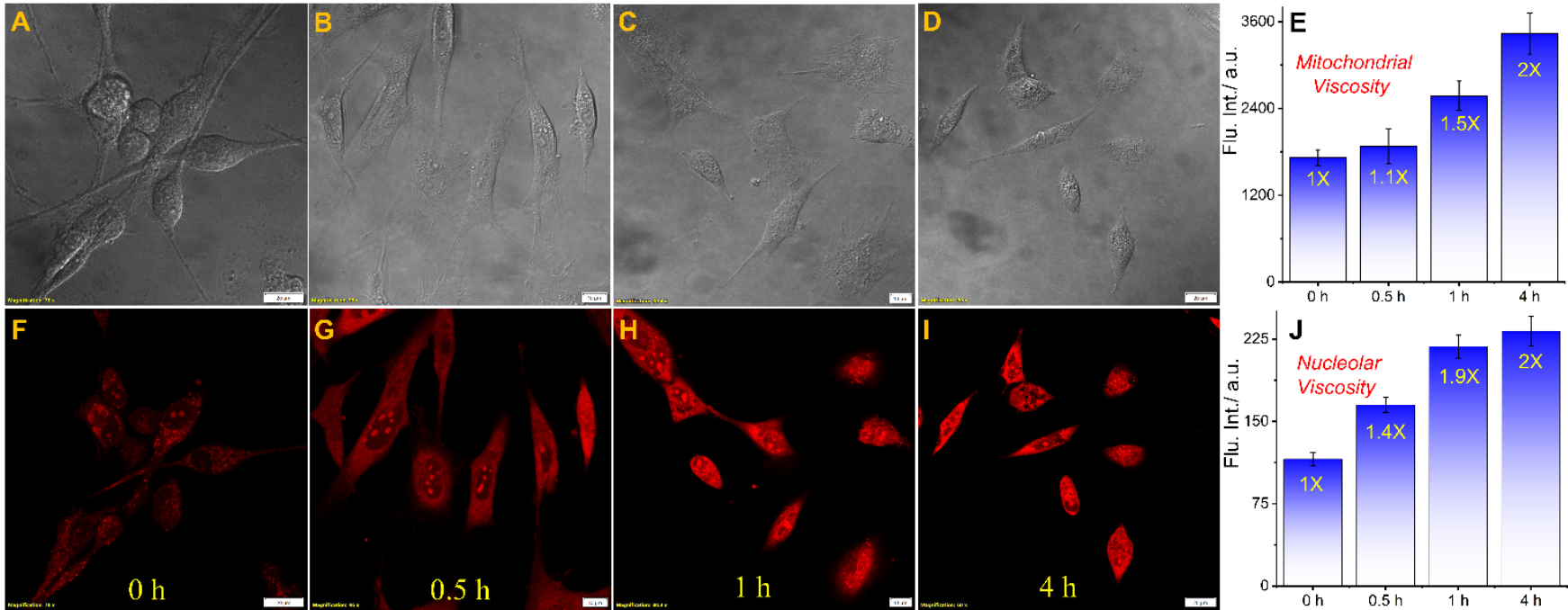

Figure 3. Confocal microscopy images of $\mathrm{U}-87 \mathrm{MG}$ cells stained with $5 \mu \mathrm{M} \mathbf{Q R}$ and treated with monensin to monitor viscosity changes at different time intervals. (A-D) DIC images; incubated with (F) QR only, and with $10 \mu \mathrm{M}$ monensin for (G) $30 \mathrm{~min},(\mathbf{H})$ $1 \mathrm{~h},(\mathrm{I}) 4 \mathrm{~h}$; bar plot shows (E) the mitochondrial viscosity increment, and (J) the nucleolus viscosity increment (Scale bar: $10 \mu \mathrm{m}$ ) (quantification performed considering at least 10 different ROIs for each time-points).

co-incubated with QR in U-87 MG cells, and Pearson's correlation coefficient was found to be o.89 suggesting fast and efficient localization of QR in mitochondria (Figure 2A-E). Furthermore, other commercially available organelle targeting dyes i.e., ER-Tracker Green (Figure 2F-I) and LysoTracker Green (Figure $\mathbf{2 K - N}$ ) were taken for costaining with QR to validate if there is any non-specific localization. Upon analyzing the colocalization results, in both cases, Pearson's co-efficient coefficient was found to be quite less (Figure 2J \& 2O). Even though the mitochondria were lit up by QR staining, Pearson's coefficient wasn't as higher as we had anticipated. A strong fluorescence signal was also detected from the nucleolus upon staining with $\mathbf{Q R}$, as small spherical compartments inside the nucleus could clearly be distinguished. Therefore, $\mathbf{Q R}$ proved to be an efficient dual-mode mitochondrial and nucleolus marker and can be useful to simultaneously monitor viscosity changes in both of these cellular compartments.

Mapping intracellular microviscosity. In order to explore if $\mathbf{Q R}$ could detect the microviscosity changes, we used a well-known ionophore monensin that facilitates the transport of $\mathrm{Na}^{+}$and $\mathrm{K}^{+}$ions between intracellular and extracellular spaces causing viscosity enhancement by swelling of mitochondria through the disruption of ionic
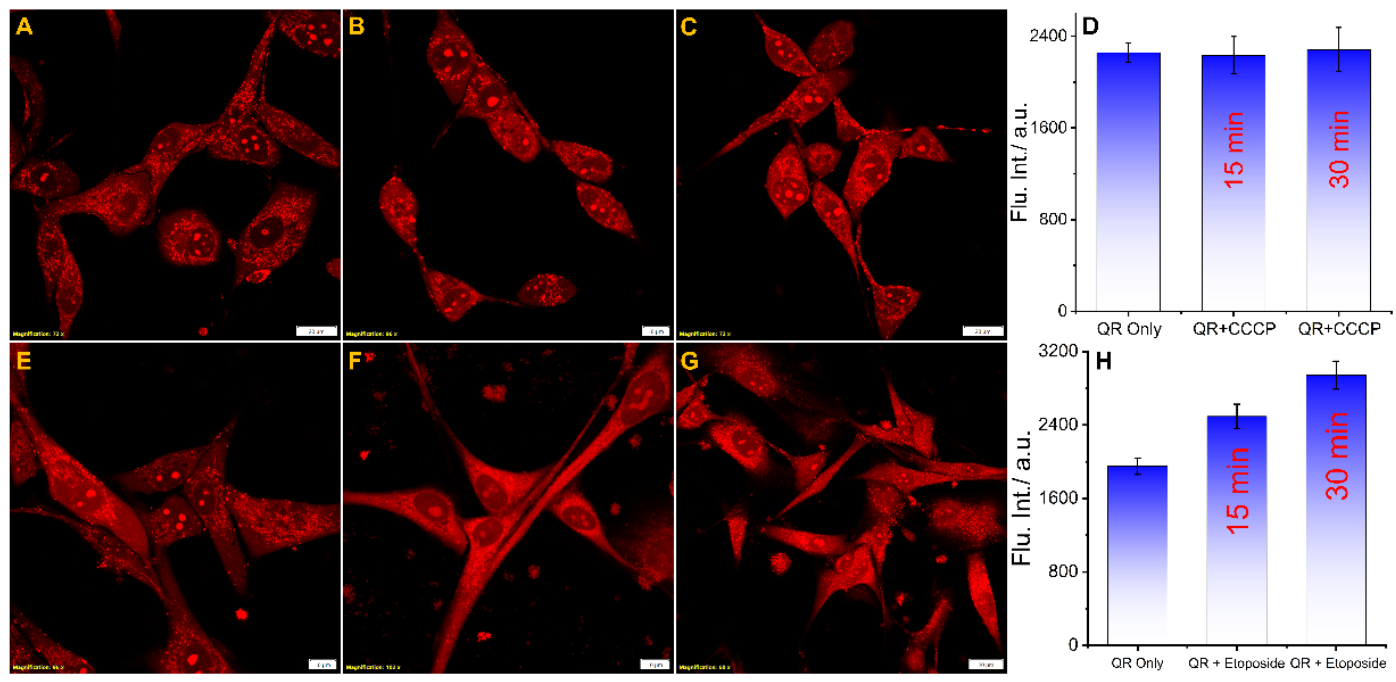

Figure 4. Confocal microscopy images of U-87 MG cells stained with $5 \mu \mathrm{M}$ QR and treated with CCCP and etoposide; (A) \& (E) incubated with QR only, treated with $20 \mu \mathrm{M} \mathrm{CCCP}$ for (B) $15 \mathrm{~min}$, (C) $30 \mathrm{~min}$, (D) bar plot shows no change in the intensity even after membrane potential uncoupling; treated with $50 \mu \mathrm{M}$ etoposide for (F) $15 \mathrm{~min}, \mathbf{G}) 30$ min, and (H) bar plot shows the viscosity enhancement upon induced apoptosis. (Scale bar: $10 \mu \mathrm{m}$ ) (quantification was performed considering at least 1o different ROIs for each time-points) 
balance. ${ }^{4-46}$ The living U-87 MG cells were treated with 10 $\mu \mathrm{M}$ monensin and incubated for $30 \mathrm{~min}, 1 \mathrm{~h}$, and $4 \mathrm{~h}$ before staining with $5 \mu \mathrm{M}$ QR. (Figure $3 \mathbf{A}-\mathbf{D}, \mathbf{F}-\mathbf{I}$ ). The post-staining analysis showed a gradual increase in the fluorescence intensity with a longer incubation period as the mitochondrial viscosity shoots up to 2-fold after 4 h (Figure 3 E). A similar analysis was performed considering the ROIs from nucleolus showed a 2-fold viscosity increment (Figure 3J). Noticeably, the viscosity increment of the nucleolus was faster compared to mitochondria.

Does the membrane potential drive the localization? Carbonyl cyanide $m$-chlorophenylhydrazone (CCCP) is a typical uncoupler of oxidative phosphorylation in mitochondria, which is widely used in studies on mitochondrial uncoupling. ${ }^{47-49}$ Hence, the mitochondrial localization depends on the disruption of the membrane potential. Therefore, we treated the U-87 MG cells with $20 \mu \mathrm{M}$ CCCP and incubated them for two different periods (Figure $4 \mathbf{A}-\mathbf{D}$ ). However, there was no change in the fluorescence intensity coming from the mitochondrial compartment, and localization was observed with the same efficiency. Additionally, the mitochondrial morphology remained unaltered even after 30 min treatment of CCCP dismissing any possibility of non-specific localization of QR upon mitochondrial membrane depolarization. This points towards the fact that the localization of $\mathbf{Q R}$ is independent of the mitochondrial membrane potential (MMP).

Effect of induced apoptosis and cell cycle arrest on microviscosity. Further experiments were carried out to monitor mitochondrial viscosity change upon induced apoptosis. Etoposide increases the reactive oxygen species production causing mitochondrial damage which eventually results in increased viscosity. ${ }^{33,}{ }^{50} \mathrm{U}-87 \mathrm{MG}$ cells were treated with $50 \mu \mathrm{M}$ etoposide and incubated for $15 \mathrm{~min}$ and $30 \mathrm{~min}$, and a significant enhancement of mitochondrial viscosity was observed (Figure ${ }_{4} \mathbf{E}-\mathbf{H}$ ). This suggests the utility of the probe to monitor mitochondrial viscosity changes in different homeostatic and non-homeostatic conditions. We have also explored the possibility of microviscosity changes upon cell cycle arrest and thereby being able to monitor it. Tunicamycin (Tm) is a well-known antibiotic that inhibits the glycosylation of the nascent proteins causing deficiency of the biologically important glycoproteins in the cell. ${ }^{51-53}$ It is also widely used for the activation of unfolded protein response (UPR) and induces endoplasmic reticulum stress. ${ }^{54-56}$ Eventually, it acts as the primary agent for the accumulation of cell cycle regulator protein $\mathrm{p} 27$, essential for $\mathrm{G}_{1} / \mathrm{S}$ phase progression. ${ }^{57-58}$ Interestingly, the ER stress induction also causes a significant enhancement in the protein level in the mitochondrial membrane, encouraging us to inspect any change in the microviscosity. As the localization of $\mathbf{Q R}$ is mitochondrial potential-independent, the Tm treated $\left(1 \mu \mathrm{g} \cdot \mathrm{mL}^{-1}\right)$ cells were stained with $\mathbf{Q R}$ and the fluorescence signals were measured thereafter. Much to our delight, the average intensity measured from the mitochondrial compartment was significantly higher post-Tm treatment for $24 \mathrm{~h}$ justifies our rationale (Figure 5A-E). Therefore, we believe, $\mathbf{Q R}$ finds its way precisely to the mitochondrial membrane. We, further, intuitively measured the intensity coming from nucleolus under the same experimental conditions. Based on the average signal intensity calculated from the different nucleolus ROIs, it was evident that nucleolar microviscosity also increased by Tm. Even though no reports directly correlate the effect of Tm on nucleolar microviscosity, we believe it plays a definite role in it. At the beginning of mitosis, the nucleolus disassembles and gets dispersed in different parts of the cell while reassembly takes place in the telophase and early $G_{1}$ phase. It is well-known that condensation of the ribosome assembly factor occurs after Tm addition to the cells leading to the enhancement of the fluorescence signal. ${ }^{59^{-60}}$ In homeostatic conditions, such condensation results in the increased molecular density of the nucleolus. ${ }^{59^{-61}}$ However, the extent of signal enhancement, and thereby the microviscosity, is not too high (Figure ${ }_{5} \mathbf{E}$ ). This can be explained by the effect of another retrograding factor in this aspect. The synthesis of some glycoproteins brings about the natural decrease of nucleolar size. Nonetheless, such a natural decrease is delayed

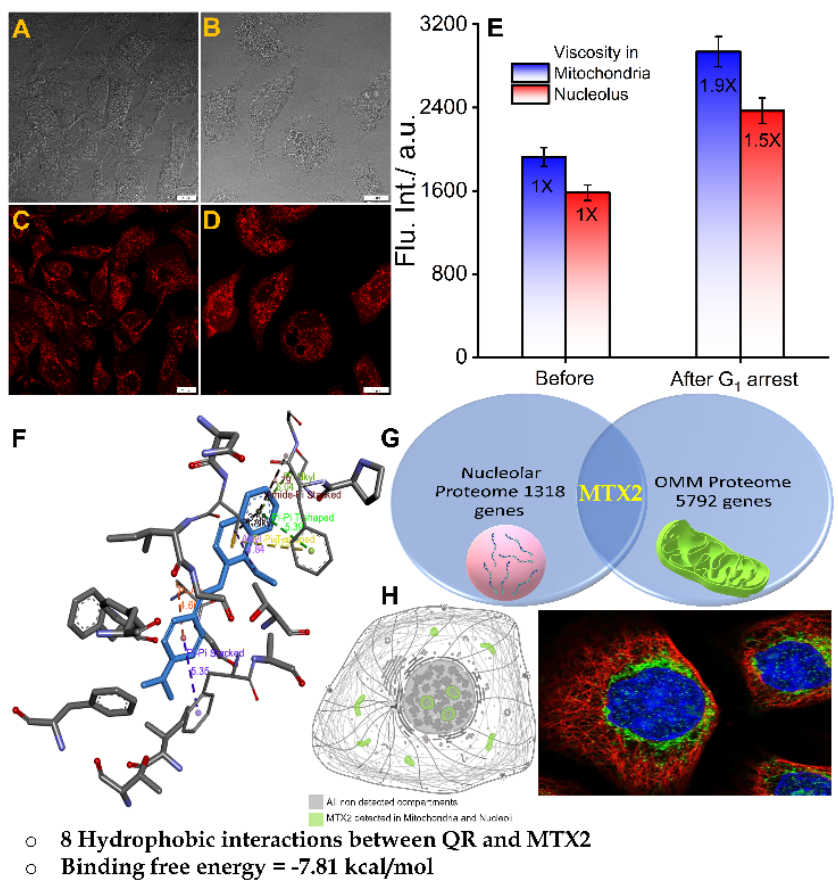

Figure 5. (Top) Confocal microscopy images of U-87 MG cells stained with $5 \mu \mathrm{M}$ QR (A) and (C) before, (B), and (D) after G1 phase cell cycle arrest induced by $1 \mu \mathrm{g} . \mathrm{ml}-1$ tunicamycin for $24 \mathrm{~h}$; (E) bar plot shows the enhancement of mitochondrial and nucleolar viscosity after $\mathrm{G}_{1}$ arrest. (Scale bar: $20 \mu \mathrm{m}$ ). (Bottom) (F) Schematic representation of the metaxin-2 (MTX2) protein that is localized in the outer mitochondrial membrane (OMM) and nucleoli. The molecular docking shows strong hydrophobic interactions of QR with MTX2, (G) The human proteome atlas shows that MTX2 is localized to the mitochondria and nucleoli; the green parts (mitochondria and nucleolus) denote the presence of MTX2, (H) The subcellular location is supported by literature; immunofluorescent staining of human cell line A-431 shows localization to nucleoli \& mitochondria. $^{65}$ 
after Tm treatment due to inhibition of glycoprotein synthesis. $^{62}$

Mitochondria-nucleolus communication: The responsible factor. As these findings suggest, such simultaneous dual organelle localization of QR leads us to look for a common factor. QR bears a positive charge and it is unlikely to cross the outer mitochondrial membrane (OMM) as the intermembrane space is rich in $\mathrm{H}^{+}$ions. Hence, our observations could only be rationalized if $\mathbf{Q R}$ binds to a specific protein that is present in both these organelles. Therefore, we have searched for proteins that are common between the OMM proteome and the nucleoli proteome. ${ }^{63-}$ ${ }^{64}$ We restricted our search to only high confidence hits and found only a single protein that was common to both proteomes namely metaxin-2 or MTX2 (Figure ${ }_{5} \mathbf{G}$ ). This was further verified by appropriate annotation of cellular localization from the human proteome atlas. ${ }^{65} \mathrm{~A}$ molecular docking study of QR using PatchDock server ${ }^{66}$ with default parameters suggested a strong interaction with -7.81 $\mathrm{kcal} / \mathrm{mol}$ binding energy mediated by 8 hydrophobic interactions (Figure $\mathbf{5} \mathbf{F}$ and Figure $\mathbf{S}_{\mathbf{5}}$ ). The analysis and visualization of the binding interactions were studied using BIOVIA Discovery Studio Visualizer. MTX2, a 263 amino acid residue protein, is a part of the metaxin protein family that is found in OMM. MTX2 carries genetic information and plays a major role in protein transport into the mitochondria ${ }^{18,67-69}$. Deficiency of MTX2 leads to fragmentation of mitochondrial network, alteration of oxidative phosphorylation, the resistance of tumor necrosis factor-induced $(\mathrm{TNF}-\alpha)$ cell death while increasing the senescence. ${ }^{70-71}$ Alongside, such deficiency results in morphological abnormalities of the nucleus that has been ultimately

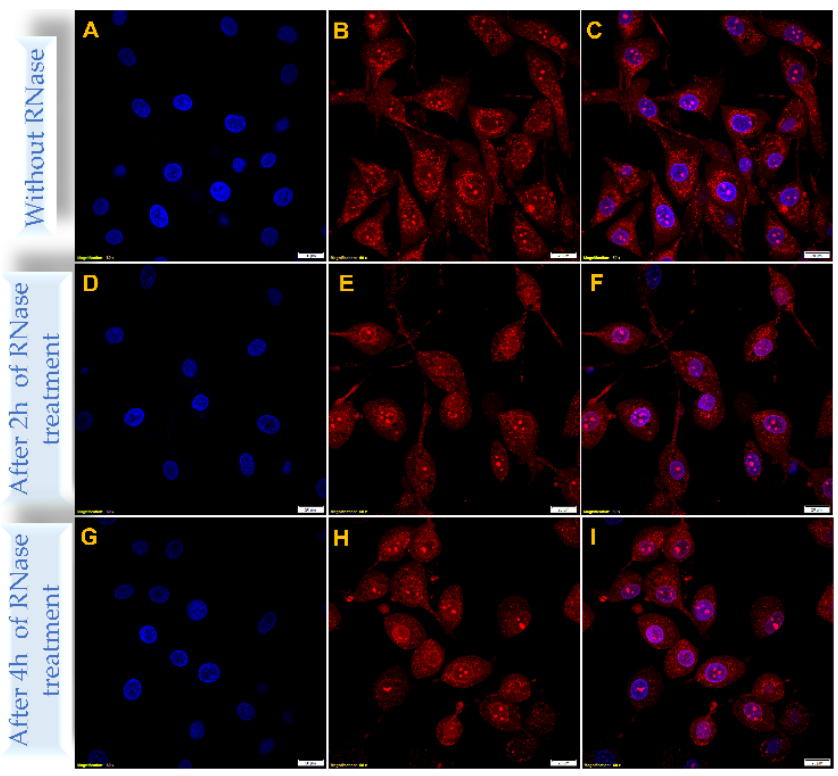

Figure 6. Confocal microscopy images of U-87 MG cells stained with $8 \mu \mathrm{M}$ Hoechst 33342 (left panel), $5 \mu \mathrm{M}$ QR (middle panel), and merge images of blue and red channel (right panel); intracellular localization (A-C) before RNase treatment, (D-F) after $100 \mu \mathrm{M}$ RNase treatment for $2 \mathrm{~h}$, and (G-I) $100 \mu \mathrm{M}$ RNase treatment for $4 \mathrm{~h}$. (Scale bar: $20 \mu \mathrm{m}$ ) linked to mandibuloacral dysplasia, a rare genetic disorder. $^{71}$ More importantly, the literature suggests that MTX2 is also localized in the nucleolus ${ }^{65}$. As all proteins located in the mitochondrial outer membrane are synthesized by cytosolic ribosomes and encoded in the nucleus while ribosome biogenesis occurs in nucleoli, the connection between nucleoli and mitochondria can be well-ascertained.

RNA digestion: Unaltered nucleolus staining. Intending to have further confirmation of the fact that QR binds to a common nucleolar and outer mitochondrial membrane protein MTX2, and not with RNA in the nucleolus, we opted for an RNA digestion experiment. Small molecular cationic fluorescent probes are prone to interact with the phosphate backbone and nucleotide of RNA ${ }^{72-73}$. Therefore, live U-87 MG cells, were treated with $100 \mu \mathrm{M}$ DNase free RNase A for $4 \mathrm{~h}$ and co-stained with $8 \mu \mathrm{M}$ Hoechst 33342 and $5 \mu \mathrm{M}$ QR. The cells were imaged for different periods of RNase A treatment ( $2 \mathrm{~h}$ and $4 \mathrm{~h}$ ) to check for any alteration in the nucleolar staining (Figure 6). However, the fluorescence signal from the nucleolar compartment remained unaffected even after $4 \mathrm{~h}$ of RNase treatment validating our finding of QR-MTX2 interaction. Therefore, upon binding to one of the most important OMM proteins, $\mathbf{Q R}$ establishes itself as a marker for deciphering the mitochondria-nucleolus communication in live cells.

Staining E. coli with QR: Presence of Metaxin-like proteins. Identification of the specific interaction that enables QR to simultaneously stain mitochondria and nucleolus led us to further check the possibility of staining bacteria. Metaxin like proteins are also present in bacteria, even though, the metaxin family was initially considered to be found in vertebrates ${ }^{74}$. Hence, we have treated $E$. coli (OP50 strain) with $10 \mu \mathrm{M} \mathbf{Q R}$ and imaged them in a timedependent manner (Figure S6). Interestingly, as seen under the microscope, the number of stained bacteria increased with a longer incubation time with the probe.

\section{CONCLUSIONS}

In conclusion, we introduce a well-known indicator dye, Quinaldine red as a microviscosity sensor and organelle communication marker. The spectroscopic investigations show that the emission enhancement caused by the inhibition of ICT in the probe forms a basis for its sensitivity towards micro-environmental viscosity. Notably, the probe was able to sense solvent viscosity over a very wide range from 1.1 to $480.6 \mathrm{cP}$, which broadens its future bioimaging applications. Intracellular localization studies in live U-87 MG cells revealed its accumulation in both mitochondria and nucleolus and its permeability remained unperturbed with mitochondrial depolarization. This stable, straightforward, and non-toxic dye may have future applications in effectively quantifying viscosity in the abovementioned organelles. Moreover, we have monitored and rationalized the enhancement of the microviscosity in both mitochondria and nucleolus in homeostatic and non-homeostatic conditions. The live-cell imaging experiments 
demonstrate how the local microenvironment behaves in both these organelles during induced apoptosis and cellcycle arrest. The probe's ability to sense microviscosity and simultaneous dual-organelle localization were intuitively employed to study the communication between mitochondria and nucleolus. We have identified a protein, metaxin2 , that is present in the outer mitochondria membrane and the nucleolus, responsible for such communication. We anticipate these findings would open up a plethora of directions towards a better understanding of the inter-related behavior of mitochondria and nucleolus and thereby further helping to decipher inter-organelle communications.

\section{AUTHOR INFORMATION}

\section{Corresponding Author}

*akoner@iiserb.ac.in

\section{Author Contributions}

TD: Live-cell imaging, bacterial imaging, molecular docking, data curation, and analysis, manuscript writing; SD: spectroscopic experiments, manuscript writing; IG: Proteome analysis; ALK: data curation, project design.

\# TD and SD contributed equally to this work.

\section{ACKNOWLEDGMENTS}

We thank the instrumentation and infrastructural facility at the Department of Chemistry of IISERB. The financial support from IISERB (ALK) and IITD (IG) is gratefully acknowledged. We acknowledge the Department of Science and Technology (DST), INDIA, for the FIST support (SR/FST/LSI-643/2015) for the live-cell imaging facility.

\section{Notes}

The authors declare no conflict of financial interest.

\section{REFERENCES}

1. Bravo-Sagua, R.; Torrealba, N.; Paredes, F.; Morales, P. E.; Pennanen, C.; Lopez-Crisosto, C.; Troncoso, R.; Criollo, A.; Chiong, M.; Hill, J. A.; Simmen, T.; Quest, A. F.; Lavandero, S., Organelle communication: Signaling crossroads between homeostasis and disease. Int. J. Biochem. Cell Biol. 2014, 50, 55-59.

2. Gao, Q.; Goodman, J. M., The lipid droplet-a wellconnected organelle. Front. Cell Dev. Biol. 2015, 3.

3. Lujan, P.; Angulo-Capel, J.; Chabanon, M.; Campelo, F., Interorganelle communication and membrane shaping in the early secretory pathway. Curr. Opin. Cell Biol. 2021, 71, 95-102.

4. Xu, J.; Zhang, Y.; Yu, H.; Gao, X.; Shao, S., MitochondriaTargeted Fluorescent Probe for Imaging Hydrogen Peroxide in Living Cells. Anal. Chem. 2016, 88 (2), 1455-1461.

5. Scorrano, L.; De Matteis, M. A.; Emr, S.; Giordano, F.; Hajnoczky, G.; Kornmann, B.; Lackner, L. L.; Levine, T. P.; Pellegrini, L.; Reinisch, K.; Rizzuto, R.; Simmen, T.; Stenmark, H.; Ungermann, C.; Schuldiner, M., Coming together to define membrane contact sites. Nat. Commun. 2019, 10, 1-11.

6. Xia, M. F.; Zhang, Y. Z.; Jin, K.; Lu, Z. T.; Zeng, Z. Y.; Xiong, W., Communication between mitochondria and other organelles: a brand-new perspective on mitochondria in cancer. Cell Biosci. 2019, 9.

\section{ASSOCIATED CONTENT}

Supporting Information

This material is available free of charge via the Internet at http://pubs.acs.org at DOI:

Supporting figures and experimental procedures (PDF)

7. Raturi, A.; Simmen, T., Where the endoplasmic reticulum and the mitochondrion tie the knot: The mitochondriaassociated membrane (MAM). Biochim Biophys Acta Mol. Cell Res. 2013, 1833 (1), 213-224.

8. Tait, S. W.; Green, D. R., Mitochondria and cell signalling. J Cell Sci. 2012, 125, 807-15.

9. Rajagopal, M. C.; Brown, J. W.; Gelda, D.; Valavala, K. V.; Wang, H.; Llano, D. A.; Gillette, R.; Sinha, S., Transient heat release during induced mitochondrial proton uncoupling. Commun. Biol. 2019, 2 (1), 279.

10. Calvo, S. E.; Mootha, V. K., The Mitochondrial Proteome and Human Disease. Annu. Rev. Genomics Hum. Genet. 2010, 11 (1), 25-44.

11. Dickinson, B. C.; Srikun, D.; Chang, C. J., Mitochondrial-targeted fluorescent probes for reactive oxygen species. Curr. Opin. Chem. Biol. 2010, 14 (1), 50-56.

12. Ma, C.; Xia, F.; Kelley, S. O., Mitochondrial Targeting of Probes and Therapeutics to the Powerhouse of the Cell. Bioconjug. Chem. 2020, 31 (12), 2650-2667.

13. Cheng, D.; Pan, Y.; Wang, L.; Zeng, Z. B.; Yuan, L.; Zhang, X. B.; Chang, Y. T., Selective Visualization of the Endogenous Peroxynitrite in an Inflamed Mouse Model by a Mitochondria-Targetable Two-Photon Ratiometric Fluorescent Probe. J Am Chem Soc 2017, 139 (1), 285-292.

14. Zheng, A.; Liu, H.; Gao, X.; Xu, K.; Tang, B., A Mitochondrial-Targeting Near-Infrared Fluorescent Probe for Revealing the Effects of Hydrogen Peroxide And Heavy Metal Ions on Viscosity. Anal. Chem. 2021, 93 (26), 9244-9249.

15. Jiao, X.; Li, Y.; Niu, J.; Xie, X.; Wang, X.; Tang, B., SmallMolecule Fluorescent Probes for Imaging and Detection of Reactive Oxygen, Nitrogen, and Sulfur Species in Biological Systems. Anal. Chem. 2018, 90 (1), 533-555.

16. Robson, J. A.; Kubánková, M.; Bond, T.; Hendley, R. A.; White, A. J. P.; Kuimova, M. K.; Wilton-Ely, J. D. E. T., Simultaneous Detection of Carbon Monoxide and Viscosity Changes in Cells. Angew. Chem. Int. Ed. 2020, 59 (48), 21431-21435.

17. Wisnovsky, S.; Lei, Eric K.; Jean, Sae R.; Kelley, Shana O., Mitochondrial Chemical Biology: New Probes Elucidate the Secrets of the Powerhouse of the Cell. Cell Chem. Biol. 2016, 23 (8), 917-927.

18. Lee, C.; Nam, J. S.; Lee, C. G.; Park, M.; Yoo, C.-M.; Rhee, H.-W.; Seo, J. K.; Kwon, T.-H., Analysing the mechanism of mitochondrial oxidation-induced cell death using a multifunctional iridium(III) photosensitiser. Nat. Commun. 2021, $12(1), 1-11$.

19. He, L.; Yang, Y.; Lin, W., Rational Design of a Rigid Fluorophore-Molecular Rotor-Based Probe for High Signal-toBackground Ratio Detection of Sulfur Dioxide in Viscous System. Anal. Chem. 2019, 91 (23), 15220-15228. 
2o. Haidekker, M. A.; Theodorakis, E. A., Molecular rotors-fluorescent biosensors for viscosity and flow. Org. Biomol. Chem. 2007, 5 (11), 1669-78.

21. Sasaki, S.; Drummen, G. P. C.; Konishi, G.-i., Recent advances in twisted intramolecular charge transfer (TICT) fluorescence and related phenomena in materials chemistry. $J$. Mater. Chem. C 2016, 4 (14), 2731-2743.

22. Wagner, N.; Stephan, M.; Höglinger, D.; Nadler, A., A Click Cage: Organelle-Specific Uncaging of Lipid Messengers. Angew. Chem.Int. Ed. 2018, 57 (40), 13339-13343.

23. Theodossiou, T. A.; Sideratou, Z.; Katsarou, M. E.; Tsiourvas, D., Mitochondrial Delivery of Doxorubicin by Triphenylphosphonium-Functionalized Hyperbranched Nanocarriers Results in Rapid and Severe Cytotoxicity. Pharm. Res. 2013, 30 (11), 2832-2842.

24. Biswas, S.; Dodwadkar, N. S.; Piroyan, A.; Torchilin, V. P., Surface conjugation of triphenylphosphonium to target poly(amidoamine) dendrimers to mitochondria. Biomaterials 2012, 33 (18), 4773-4782.

25. Smith, R. A. J.; Porteous, C. M.; Coulter, C. V.; Murphy, M. P., Selective targeting of an antioxidant to mitochondria. Eur: J. Biochem. 1999, 263 (3), 709-716.

26. Filipovska, A.; Kelso, G. F.; Brown, S. E.; Beer, S. M.; Smith, R. A. J.; Murphy, M. P., Synthesis and Characterization of a Triphenylphosphonium-conjugated Peroxidase Mimetic: INSIGHTS INTO THE INTERACTION OF EBSELEN WITH MITOCHONDRIA*. J. Biol. Chem. 2005, 280 (25), 24113-24126.

27. Kelso, G. F.; Porteous, C. M.; Coulter, C. V.; Hughes, G.; Porteous, W. K.; Ledgerwood, E. C.; Smith, R. A.; Murphy, M. P., Selective targeting of a redox-active ubiquinone to mitochondria within cells: antioxidant and antiapoptotic properties. J. Biol. Chem. 2001, 276 (7), 4588-4596.

28. Baracca, A.; Sgarbi, G.; Solaini, G.; Lenaz, G., Rhodamine 123 as a probe of mitochondrial membrane potential: evaluation of proton flux through Fo during ATP synthesis. Biochim. Biophys. Acta, Bioenerg. 2003, 1606 (1), 137-146.

29. Yang, Z.; He, Y.; Lee, J.-H.; Park, N.; Suh, M.; Chae, W.S.; Cao, J.; Peng, X.; Jung, H.; Kang, C.; Kim, J. S., A Self-Calibrating Bipartite Viscosity Sensor for Mitochondria. J. Am. Chem. Soc. 2013, 135 (24), 9181-9185.

30. Chen, H.; Wang, J.; Feng, X.; Zhu, M.; Hoffmann, S.; Hsu, A.; Qian, K.; Huang, D.; Zhao, F.; Liu, W.; Zhang, H.; Cheng, Z., Mitochondria-targeting fluorescent molecules for high efficiency cancer growth inhibition and imaging. Chem. Sci. 2019, 10 (34), 7946-7951.

31. Tan, X.; Luo, S.; Long, L.; Wang, Y.; Wang, D.; Fang, S.; Ouyang, Q.; Su, Y.; Cheng, T.; Shi, C., Structure-Guided Design and Synthesis of a Mitochondria-Targeting Near-Infrared Fluorophore with Multimodal Therapeutic Activities. Adv. Mater. 2017, 29 (43), 1704196.

32. Zhang, G.; Sun, Y.; He, X.; Zhang, W.; Tian, M.; Feng, R.; Zhang, R.; Li, X.; Guo, L.; Yu, X.; Zhang, S., Red-Emitting Mitochondrial Probe with Ultrahigh Signal-to-Noise Ratio Enables High-Fidelity Fluorescent Images in Two-Photon Microscopy. Anal. Chem. 2015, 87 (24), 12088-12095.

33. Jimenez-Sanchez, A.; Lei, E. K.; Kelley, S. O., A Multifunctional Chemical Probe for the Measurement of Local Micropolarity and Microviscosity in Mitochondria. Angew. Chem. Int. Ed. 2018, 57 (29), 8891-8895.

34. Caragine, C. M.; Haley, S. C.; Zidovska, A., Nucleolar dynamics and interactions with nucleoplasm in living cells. elife 2019, 8 .

35. Boisvert, F. M.; van Koningsbruggen, S.; Navascues, J.; Lamond, A. I., The multifunctional nucleolus. Nat. Rev. Mol. Cell Biol. 2007, 8 (7), 574-585.

36. Cogan, E. B.; Birrell, G. B.; Griffith, O. H., A RoboticsBased Automated Assay for Inorganic and Organic Phosphates. Anal. Biochem. 1999, 271 (1), 29-35.
37. Dutta, T.; Chandra, F.; Koner, A. L., A rapid, naked-eye detection of hypochlorite and bisulfite using a robust and highlyphotostable indicator dye Quinaldine Red in aqueous medium. Spectrochim. Acta A Mol. Biomol. Spectrosc. 2018, 191, 217-220.

38. Cai, Q.; Wang, C.; Zhou, J.; Luo, F.; Lin, Z.; Guo, L.; Qiu, B.; Chen, G., Terminal protection G-quadruplex-based turn-on fluorescence biosensor for $\mathrm{H}_{5} \mathrm{~N}_{1}$ antibody. Anal. Methods 2012, 4 (10), 3425-3428.

39. Koyama, Y.; Carey, P. R.; Long, R. A.; Martin, W. G.; Schneider, H., A resonance Raman and electronic absorption probe of membrane energization. Quinaldine red in cells of Streptococcus faecalis. J. Biol. Chem. 1979, 254 (20), 10276-10285.

40. Murphy, M. P., Targeting lipophilic cations to mitochondria. Biochim. Biophys. Acta Bioenerg. 20o8, 1777 (7-8), 1028-1031.

41. Leo, A.; Hansch, C.; Elkins, D., Partition Coefficients and Their Uses. Chem. Rev. 1971, 71 (6), 525-616.

42. Jiang, N.; Fan, J.; Zhang, S.; Wu, T.; Wang, J.; Gao, P.; Qu, J.; Zhou, F.; Peng, X., Dual mode monitoring probe for mitochondrial viscosity in single cell. Sens. Actuators B Chem. 2014, 190, 685-693.

43. Kuimova, M. K., Mapping viscosity in cells using molecular rotors. Phys. Chem. Chem. Phys. 2012, 14 (37), 1267112686.

44. Kaushik, V.; Yakisich, J. S.; Kumar, A.; Azad, N.; Iyer, A. K. V., Ionophores: Potential Use as Anticancer Drugs and Chemosensitizers. Cancers 2018, 10 (10).

45. Zhan, J. T.; Geng, C.; Hao, X. Y.; Song, W. H.; Lin, W. Y., A near-infrared fluorescent probe for monitoring viscosity in living cells, zebrafish and mice. New J. Chem. 2021, 45 (8), 37783782 .

46. Liang, Y.; Zhao, Y. P.; Lai, C. F.; Zou, X.; Lin, W. Y., A coumarin-based TICT fluorescent probe for real-time fluorescence lifetime imaging of mitochondrial viscosity and systemic inflammation in vivo. J. Mater. Chem. B 2021.

47. Ganote, C. E.; Armstrong, S. C., Effects of CCCP-induced mitochondrial uncoupling and cyclosporin A on cell volume, cell injury and preconditioning protection of isolated rabbit cardiomyocytes. J. Mol. Cell Cardiol. 2003, 35 (7), 749-59.

48. Padman, B. S.; Bach, M.; Lucarelli, G.; Prescott, M.; Ramm, G., The protonophore CCCP interferes with lysosomal degradation of autophagic cargo in yeast and mammalian cells. Autophagy 2013, 9 (11), 1862-75.

49. Sivandzade, F.; Bhalerao, A.; Cucullo, L., Analysis of the Mitochondrial Membrane Potential Using the Cationic JC-1 Dye as a Sensitive Fluorescent Probe. Bio Protoc. 2019, 9 (1).

5o. Jamil, S.; Lam, I.; Majd, M.; Tsai, S. H.; Duronio, V., Etoposide induces cell death via mitochondrial-dependent actions of p53. Cancer Cell Int. 2015, 15.

51. Esko, J. D.; Bertozzi, C.; Schnaar, R. L., Chemical Tools for Inhibiting Glycosylation. In Essentials of Glycobiology, Varki, A.; Cummings, R. D.; Esko, J. D.; Stanley, P.; Hart, G. W.; Aebi, M.; Darvill, A. G.; Kinoshita, T.; Packer, N. H.; Prestegard, J. H.; Schnaar, R. L.; Seeberger, P. H., Eds. Cold Spring Harbor (NY), 2015; pp 701-712.

52. Prives, J.; Bar-Sagi, D., Effect of tunicamycin, an inhibitor of protein glycosylation, on the biological properties of acetylcholine receptor in cultured muscle cells. J. Biol. Chem. 1983, 258 (3), 1775-80.

53. Luesch, H.; Paavilainen, V. O., Natural products as modulators of eukaryotic protein secretion. Nat. Prod. Rep. 2020, 37 (5), 717-736.

54. Wang, X.; Eno, C. O.; Altman, B. J.; Zhu, Y.; Zhao, G.; Olberding, K. E.; Rathmell, J. C.; Li, C., ER stress modulates cellular metabolism. Biochem. J. 2011, 435 (1), 285-96.

55. Han, X.; Zhang, X.; Li, H.; Huang, S.; Zhang, S.; Wang, F.; Shi, Y., Tunicamycin enhances the antitumor activity of 
trastuzumab on breast cancer in vitro and in vivo. Oncotarget 2015, 6 (36), 38912-25.

56. Zhang, X.; Yuan, Y.; Jiang, L.; Zhang, J.; Gao, J.; Shen, Z.; Zheng, Y.; Deng, T.; Yan, H.; Li, W.; Hou, W. W.; Lu, J.; Shen, Y.; Dai, H.; Hu, W. W.; Zhang, Z.; Chen, Z., Endoplasmic reticulum stress induced by tunicamycin and thapsigargin protects against transient ischemic brain injury: Involvement of PARK2dependent mitophagy. Autophagy 2014, 10 (10), 1801-13.

57. Lee, J.; Kim, S. S., The function of p27 KIP1 during tumor development. Exp. Mol. Med. 2009, 41 (11), 765-71.

58. Han, C.; Jin, L.; Mei, Y.; Wu, M., Endoplasmic reticulum stress inhibits cell cycle progression via induction of p27 in melanoma cells. Cell Signal 2013, 25 (1), 144-9.

59. Horigome, C.; Okada, T.; Matsuki, K.; Mizuta, K., A ribosome assembly factor Ebp2p, the yeast homolog of EBNA1binding protein 2, is involved in the secretory response. Biosci. Biotechnol. Biochem. 2008, 72 (4), 1080-6.

6o. Shirai, C.; Takai, T.; Nariai, M.; Horigome, C.; Mizuta, K., Ebp2p, the yeast homolog of Epstein-Barr virus nuclear antigen 1-binding protein 2, interacts with factors of both the 60 $\mathrm{S}$ and the $40 \mathrm{~s}$ ribosomal subunit assembly. J. Biol. Chem. 2004, 279 (24), 25353-8.

61. Tsujii, R.; Miyoshi, K.; Tsuno, A.; Matsui, Y.; Toh-e, A.; Miyakawa, T.; Mizuta, K., Ebp2p, yeast homologue of a human protein that interacts with Epstein-Barr virus Nuclear Antigen 1, is required for pre-rRNA processing and ribosomal subunit assembly. Genes Cells 200o, 5 (7), 543-553.

62. Altmann, G. G., A gradual decrease in nucleolar size with the maturation of columnar epithelial cells in the adult rat intestine under normal and various experimental conditions. $J$. Cell Sci. 1985, 77, 289-304.

63. Stenstrom, L.; Mahdessian, D.; Gnann, C.; Cesnik, A. J.; Ouyang, W.; Leonetti, M. D.; Uhlen, M.; Cuylen-Haering, S.; Thul, P. J.; Lundberg, E., Mapping the nucleolar proteome reveals a spatiotemporal organization related to intrinsic protein disorder. Mol. Syst. Biol. 2020, 16 (8).

64. Hung, V.; Lam, S. S.; Udeshi, N. D.; Svinkina, T.; Guzman, G.; Mootha, V. K.; Carr, S. A.; Ting, A. Y., Proteomic mapping of cytosol-facing outer mitochondrial and ER membranes in living human cells by proximity biotinylation. eLife 2017, 6 .

65. The Human Protein Atlas. https://www.proteinatlas.org/ENSG00000128654-MTX2/cell human.
66. Schneidman-Duhovny, D.; Inbar, Y.; Nussinov, R.; Wolfson, H. J., PatchDock and SymmDock: servers for rigid and symmetric docking. Nucleic Acids Res. 2005, 33, W363-W367.

67. Armstrong, L. C.; Komiya, T.; Bergman, B. E.; Mihara, K.; Bornstein, P., Metaxin is a component of a preprotein import complex in the outer membrane of the mammalian mitochondrion. J. Biol. Chem. 1997, 272 (10), 6510-6518.

68. Lister, R.; Carrie, C.; Duncan, O.; Ho, L. H. M.; Howell, K. A.; Murcha, M. W.; Whelan, J., Functional definition of outer membrane proteins involved in preprotein import into mitochondria. Plant Cell 2007, 19 (11), 3739-3759.

69. Zhao, Y. S.; Song, E. L.; Wang, W. J.; Hsieh, C. H.; Wang, X. N.; Feng, W.; Wang, X. M.; Shen, K., Metaxins are core components of mitochondrial transport adaptor complexes. Nat. Commun. 2021, 12 (1).

70. Wang, X. F.; Ono, K.; Kim, S. O.; Kravchenko, V.; Lin, S. C.; Han, J. H., Metaxin is required for tumor necrosis factorinduced cell death. Embo Reports 2001, 2 (7), 628-633.

71. Elouej, S.; Harhouri, K.; Le Mao, M.; Baujat, G.; Nampoothiri, S.; Kayserili, H.; Al Menabawy, N.; Selim, L.; Paneque, A. L.; Kubisch, C.; Lessel, D.; Rubinsztajn, R.; Charar, C.; Bartoli, C.; Airault, C.; Deleuze, J. F.; Rotig, A.; Bauer, P.; Pereira, C.; Loh, A.; Escande-Beillard, N.; Muchir, A.; Martino, L.; Gruenbaum, Y.; Lee, S. H.; Manivet, P.; Lenaers, G.; Reversade, B.; Levy, N.; De Sandre-Giovannoli, A., Loss of MTX2 causes mandibuloacral dysplasia and links mitochondrial dysfunction to altered nuclear morphology Nat. Commun. 2020, 11 (1), 1-14.

72. Li, H.; Li, Y. C.; Zhang, H. H.; Xu, G. Y.; Zhang, Y. L.; Liu, X. H.; Zhou, H. P.; Yang, X. Y.; Zhang, X. J.; Tian, Y. P., Watersoluble small-molecule probes for RNA based on a two-photon fluorescence "off-on" process: systematic analysis in live cell imaging and understanding of structure-activity relationships. Chem. Commun. 2017, 53 (99), 13245-13248.

73. Mukherjee, T.; Soppina, V.; Ludovic, R.; Mely, Y.; Klymchenko, A. S.; Collot, M.; Kanvah, S., Live-cell imaging of the nucleolus and mapping mitochondrial viscosity with a dual function fluorescent probe. Org. Biomol. Chem. 2021, 19 (15), 33893395 .

74. Adolph, K. W., Invertebrate Metaxins 1 and 2: Widely Distributed Proteins Homologous to Vertebrate Metaxins Implicated in Protein Import into Mitochondria. bioRxiv 2020, 2020.01.06.895979.

\section{Table of Contents artwork}

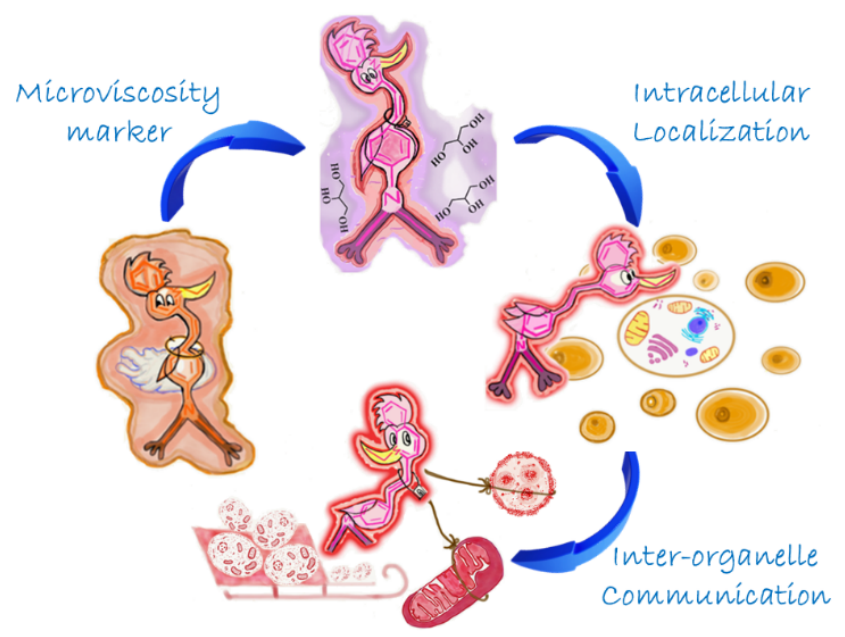

